\title{
Sarcoidosis in gastric cancer at the time of diagnosis: A case report
}

\author{
YANG JIAO ${ }^{1}$, JIE NING $^{1}$, WEN-DI ZHAO ${ }^{2}$, YAN-LI LI $^{3}$, HONG-YANG WU ${ }^{1}$ and KANG-SHENG GU ${ }^{1}$ \\ Departments of ${ }^{1}$ Oncology and ${ }^{2}$ Pathology, The First Affiliated Hospital of Anhui Medical University, Hefei, Anhui 230022; \\ ${ }^{3}$ Department of Hematology, The Second Affiliated Hospital of Anhui Medical University, Hefei, Anhui 230601, P.R. China
}

Received June 2, 2014; Accepted September 22, 2014

DOI: $10.3892 / 01.2015 .2850$

\begin{abstract}
Sarcoidosis is a multisystemic inflammatory disease that commonly affects the lungs and lymphatic system and is characterized by the formation of non-caseating granulomas. Although the association between sarcoidosis and malignant diseases has been well described, it remains controversial whether this association is merely a coincidence or the consequence of a common pathophysiological mechanism. The present study reports a rare case of sarcoidosis that was present in a patient with gastric cancer at the time of diagnosis. A 64-year-old female diagnosed with stage I gastric cancer underwent curative surgery, and the postoperative pathology of the lymph nodes revealed non-caseating granulomas. At the 4-year follow-up, the sarcoidosis remained stable, and no recurrence of cancer was identified. The present case revealed that sarcoidosis and gastric cancer may coexist simultaneously and focused on the potential advantages of histological confirmation in patients with cancer and sarcoidosis.
\end{abstract}

\section{Introduction}

Sarcoidosis is a systemic disorder of unknown etiology that is characterized by the widespread development of non-caseating epithelioid cell granulomas in multiple organ systems (1). The widely accepted pathogenic hypothesis is that sarcoidosis is promoted in genetically susceptible individuals by environmental factors (2). Non-caseating granulomas consisting of epithelioid cells and $\mathrm{T}$ lymphocytes are the characteristic lesions of sarcoidosis (2). The association between sarcoidosis and malignancy has been reported by several studies with conflicting results; this is caused by the clinical and radiographic features of the disease, which exhibit similarities to malignancies such as lymphoma or lung cancer and were occasionally in the absence of histological confirmation $(3,4)$. The present study reports a case of sarcoidosis mimicking cancer metastasis that was present at the time of the diagnosis

Correspondence to: Dr Kang-Sheng Gu, Department of Oncology, The First Affiliated Hospital of Anhui Medical University, 218 Ji Xi Road, Hefei, Anhui 230022, P.R. China

E-mail: gks63@tom.com

Key words: sarcoidosis, gastric cancer, diagnosis, pathology of gastric cancer and discusses the diagnostic process. The patient provided written informed consent.

\section{Case report}

A 64-year-old female presented to the Lujiang County Hospital (Hefei, China) with epigastric discomfort and a swallowing disorder that had persisted for more than one month. During the course of the disease, the symptoms of night sweating and weight loss were also present, however, no fever, coughing, expectoration, hemoptysis, nasal congestion, hematemesis or melena occurred. The patient had previously suffered from esophageal erosion. Gastroscopy revealed a gastric lesion that was located in the cardia, and pathological analysis of the lesion revealed high-grade intraepithelial neoplasia and local canceration. Bilateral hilar widening and enlarging of multiple lymph nodes in the mediastinum and hilum of the lung were revealed by chest X-ray and chest-abdomen computed tomography (CT), respectively. Positron emission tomography (PET)/CT revealed that the fluorodeoxyglucose metabolism of the lymph nodes in the bilateral supraclavicular, mediastinal, hilar, retroperitoneal, pelvic and inguinal regions had increased (Figs. 1 and 2). Based on these results, two types of disease were considered, lymphoma and a rare digestive tract tumor with multiple metastases. Lujiang County Hospital diagnosed the lesion as advanced gastric cancer.

In order to confirm the diagnosis and undergo treatment, the patient presented to the outpatient department of the First Affiliated Hospital of Anhui Medical University (Hefei, China). Upon physical examination, a tenacious lymph node, $\sim 1.0 \times 1.0 \mathrm{~cm}$ in size, was identified in the left supraclavicular root of the neck and several lymph nodes in the right inguinal region. No other lymph node swelling was observed in the superficial part of the body. Laboratory investigation indicated that a routine blood examination (white blood cells, $4.8 \times 10^{9}$ cells $/ 1$, normal range, $4-10 \times 10^{9}$ cells $/ 1$; hemoglobin, $115 \mathrm{~g} / 1$, normal range, $110-150 \mathrm{~g} / \mathrm{l}$; platelet count, $224 \times 10^{9}$ cells/1, normal range, $100-300 \times 10^{9}$ cells $/ 1$ ), liver (aspartate transaminase, $20 \mathrm{IU} / 1$, normal range, 0-40 IU/1; alanine transaminase, $22 \mathrm{IU} / 1$, normal range, 0-40 IU/1) and kidney function (blood urea nitrogen, $4.9 \mathrm{mmol} / 1$, normal range, 2.1-7.9 $\mathrm{mmol} / 1$; creatinine $85 \mu \mathrm{mol} / 1$, normal range, 44-133 $\mu \mathrm{mol} / \mathrm{l}$ ) tests were normal, the serum calcium level was $2.75 \mathrm{mmol} / \mathrm{l}$ (normal range, $2.25-2.75 \mathrm{mmol} / \mathrm{l}$ ) and the alkaline phosphatase level was $135 \mathrm{U} / 1$ (normal range, 25-125 U/1). Fine needle aspiration biopsy was performed on the left 


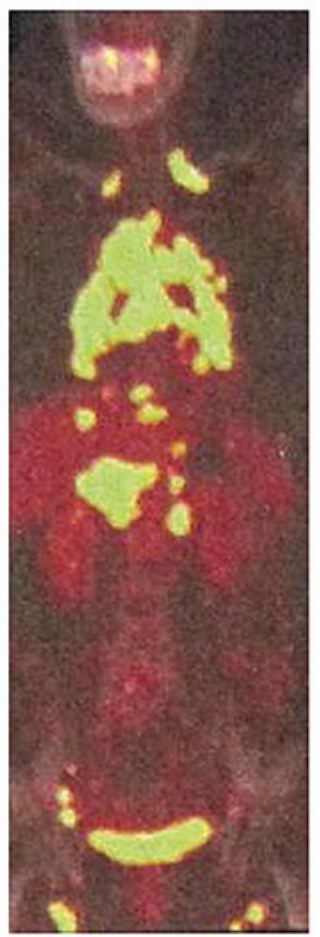

Figure 1. PET/CT images of the lymph nodes. A PET/CT scan revealing multiple areas of high fluorodeoxyglucose uptake in the bilateral supraclavicular, mediastinal, hilar, retroperitoneal, pelvic and inguinal lymph nodes. PET/CT, positron emission tomography and computed tomography.
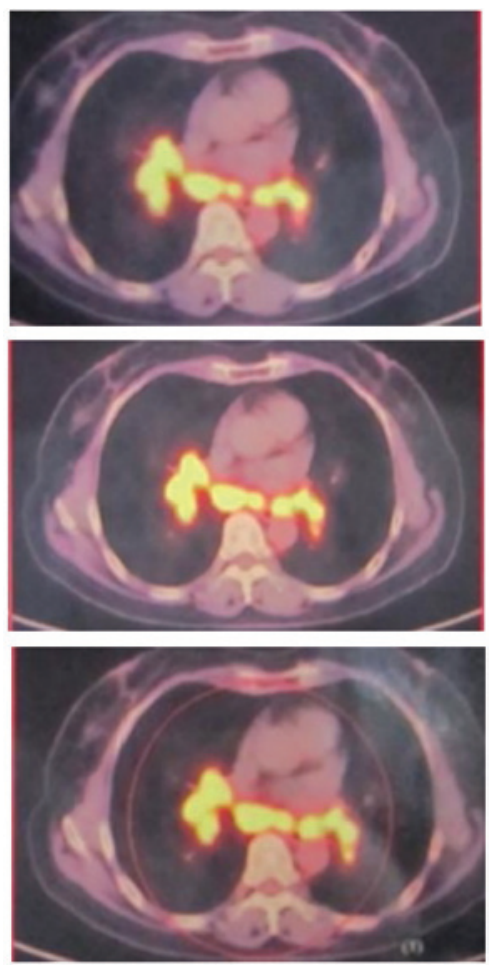

Figure 2. Positron emission tomography and computed tomography images of the mediastinal and hilar lymph nodes.

supraclavicular lymph node, and revealed lymphoreticular hyperplasia, as epithelioid cells were present. Due to the cytology results, a tuberculosis examination was performed, but the tuberculin testing exhibited a negative result. The

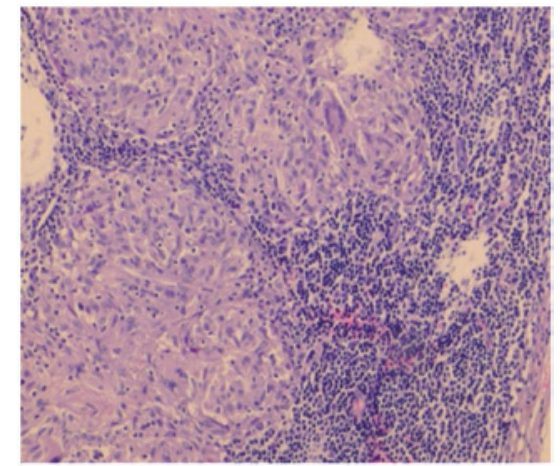

Figure 3. High-power photomicrograph of the biopsy specimen from the right inguinal lymph node revealing granulomatous infiltration with occasional multinucleated giant cells (hematoxylin and eosin stain; magnification, $\mathrm{x} 100$ ).

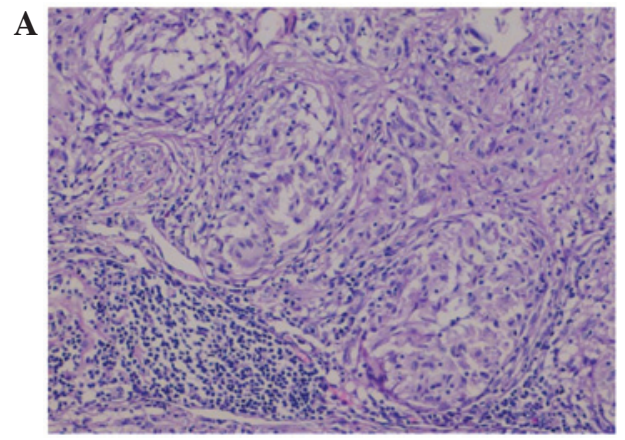

B
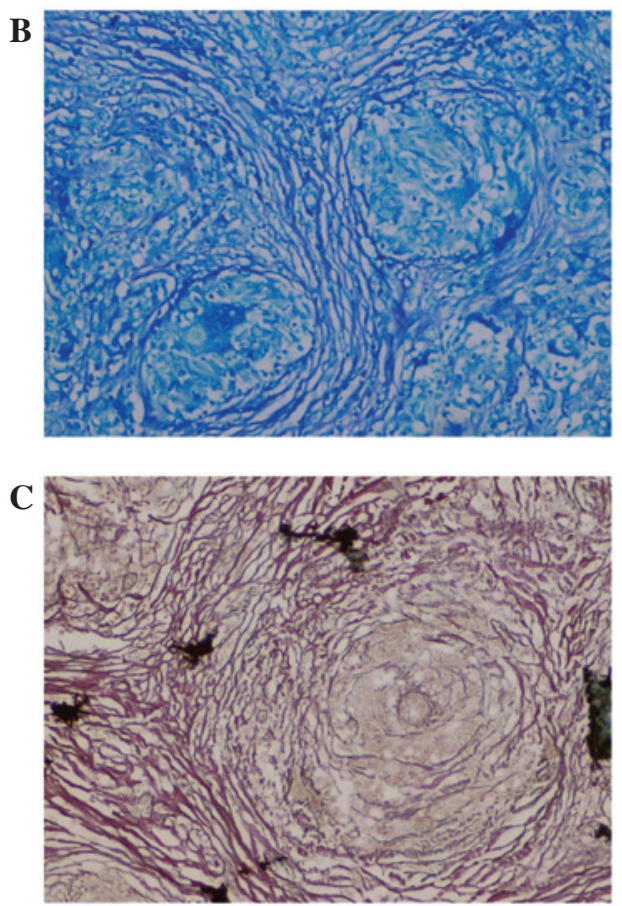

Figure 4. High-power photomicrograph of the biopsy specimen from the gastric lymph node revealing non-caseating granuloma. (A) Hematoxylin and eosin stain; magnification, x100, of the non-caseating granulomatous lesions. (B) Acid fast stain of the lesions revealing non-acid fast cells. (C) Reticular fiber-specific staining revealing numerous reticular fibers inside and around the granulomatous lesions.

biopsy of the right inguinal lymph node indicated granulomatous infiltration with occasional multinucleated giant cells (Fig. 3). 
The patient was referred to the Department of General Surgery at the first Affiliated Hospital of Anhui Medical University, having provided the appropriate informed consent, and received radical total gastrectomy combined with a Roux-en-Y procedure (5). A superficial ulcerative mass was identified inside the small curved side below the cardia and multiple enlarged lymph nodes were identified around the stomach and liver duodenum ligament, the largest of which was $2.5 \times 2.0 \mathrm{~cm}$ in diameter. The postoperative pathology indicated erosive medium differentiated adenocarcinoma in the lesser gastric curvature, $\sim 2.0 \times 1.5 \mathrm{~cm}$ in diameter. The cancer cells had invaded the layer of muscularis mucosae and a number of cells had reached the submucosa. In total, eight lymph nodes were dissected from the lesser curvature, one from the greater curvature and 23 from other areas surrounding the gastric region. The pathology of the total 32 lymph nodes indicated the presence of non-caseating granulomas (Fig. 4A and B). Therefore, the patient was diagnosed with gastric erosive medium-differentiated adenocarcinoma (pT1N0M0) (6) and sarcoidosis (I) (1). According to the NCCN guidelines for gastric cancer staging result determination, the patient did not receive chemotherapy (7). At the four-year checkup, the sarcoidosis remained stable, and no recurrence of the cancer was identified.

\section{Discussion}

The present study reports a rare case of sarcoidosis that was present in a patient with gastric cancer at the time of diagnosis, which highlights the complexity of the diagnostic process. The present case identifies that clinical presentation and radiological findings, including $\mathrm{PET/CT}$, may not be able to differentiate between cancer metastasis and sarcoidosis.

Sarcoidosis has been reported to imitate malignant neoplasms and has been identified at the time of diagnosis and during or immediately following chemotherapy, including in cases of breast (8-10) and lung (11-13) cancer, Hodgkin's disease $(14,15)$, colorectal (16-18) and head and neck cancer (19,20), melanoma (21-23) and ovarian cancer (24). Although cases of sarcoidosis mimicking metastatic gastric cancer have been rarely reported, Konishi et al (25) reported a case of advanced gastric cancer with sarcoidosis and Blank et al (26) revealed that four patients with gastric cancer were identified in their cohort of 425 patients with sarcoidosis.

The association between sarcoidosis and malignancy remains controversial. Brincker and Wilbek (27) first reported a statistically significant increase in the incidence of malignant tumors among patients with sarcoidosis in 1974. A large Japanese study followed 1,411 patients with sarcoidosis for three years and also observed an increase in mortality from leukemia and uterine cancer, using a standardized mortality risk (28). A retrospective cohort study by Askling et al (29) analyzed two cohorts of patients with sarcoidosis and identified an increased risk of lymphoma and lung, liver, and skin cancer. Boffetta et al (30) reported that the risks of rectal, colon and kidney cancer were increased in patients with sarcoidosis in their cohort study. In addition, 1,045 out of a total of 10,037 hospitalized patients with sarcoidosis were reported to subsequently develop cancer, with a significant proportion being reported for skin (squamous cell), kidney and non-thyroid endocrine tumors and additionally for non-Hodgkin's lymphoma and leukemia (31). Furthermore, in the Medical Center of the University of Heidelberg (Heidelberg, Germany), 61 patients with malignant disease were identified in the cohort of 425 patients with sarcoidosis (26). However, other studies have hypothesized that malignancy may actually precede the diagnosis of sarcoidosis. Suen et al (32) reported six cases in which sarcoidosis was diagnosed an average of nine months following the development of malignancy and termed this phenomenon malignancy-sarcoidosis syndrome. Despite the evidence suggesting that these two entities may be linked, a number of studies have challenged the existence of an association between sarcoidosis and malignancy. Rømer et al (33) reviewed the cases of sarcoidosis and malignancy that Brincker and Wilbek (27) had presented, finding that a number of cases had been misclassified and identified no increased occurrence of malignancy in patients with sarcoidosis. The importance of misclassification was also reported by Seersholm et al (34), who identified misclassification in three out of 36 malignancies in 254 patients with sarcoidosis.

Conflicting results among these various studies are caused by the similarities in the clinical and radiological features of sarcoidosis and malignancy and occasionally, the lack of histological confirmation. PET/CT imaging is a valuable tool for the diagnosis of cancer and for monitoring cancer metastasis as it allows for the location of metabolically active malignant tissue to be determined. However, the specificity of PET/CT is hampered by non-oncological medical conditions, including sarcoidosis, Wegener's granulomatosis, chronic granulomatous disease, and mycobacterial and aspergillus infections, where glucose consumption may be observed, particularly in the cellular component of inflammatory lesions. Therefore, it is important to obtain a histological diagnosis prior to initiating antineoplastic therapy based on the imaging findings.

Sarcoidosis must be considered in differential diagnosis when a cancer patient develops diffuse lymph nodal involvement. As the currently available imaging techniques fail to formally distinguish metastasis from sarcoidosis, a pathological diagnosis must be obtained wherever possible.

\section{References}

1. Newman LS, Rose CS and Maier LA: Sarcoidosis. N Engl J Med 336: 1224-1234, 1997.

2. Wu JJ and Schiff KR: Sarcoidosis. Am Fam Physician 70: 312-322, 2004.

3. Sato Y, Sasano S, Oyama K, Sakuraba M, Onuki T and Nitta S. Lung cancer associated with sarcoidosis. Jpn J Thorac Cardiovasc Surg 51: 21-24, 2003.

4. Bouros D, Hatzakis K, Labrakis $\mathrm{H}$ and Zeibecoglou K: Association of malignancy with diseases causing interstitial pulmonary changes. Chest 121: 1278-1289, 2002.

5. Scott HW Jr and Weidner MG: Total gastrectomy with Roux-en-Y esophagojejunostomy in treatment of gastric cancer. Ann Surg 143: 682-696, 1956.

6. Sobin LH, Gospodarowicz MK and Wittekind C (eds): TNM classification of malignant tumours. 7th edition. Wiley, New York, NY, 2009.

7. NCCN Guidelines version 1.2014 Panel Members Gastric Cancer. https://www.nccn.org/store/login/login.aspx?ReturnURL=http:// www.nccn.org/professionals/physician_gls/pdf/gastric.pdf. Accessed May 30, 2014

8. Tolaney SM, Colson YL, Gill RR, et al: Sarcoidosis mimicking metastatic breast cancer. Clin Breast Cancer 7: 804-810, 2007.

9. Bush E, Lamonica D and O'Connor T: Sarcoidosis mimicking metastatic breast cancer. Breast J 17: 533-535, 2011. 
10. Shin HC, Choe JW, Ryu HS, et al: Sarcoidosis mimicking metastatic breast cancer in Korean woman with breast cancer. Breast J 20: 198-199, 2014

11. Maeda J, Ohta M, Hirabayashi $\mathrm{H}$ and Matsuda H: False positive accumulation in $18 \mathrm{~F}$ fluorodeoxyglucose positron emission tomography scan due to sarcoid reaction following induction chemotherapy for lung cancer. Jpn J Thorac Cardiovasc Surg 53: 196-198, 2005

12. Umezu H, Chida M,Inoue T, et al: Sarcoidosis development during induction chemotherapy for lung cancer mimicked progressive disease. Gen Thorac Cardiovasc Surg 58: 434-437, 2010.

13. Kim JJ, Park JK, Wang YP, Choi SH and Jo KH: Lung cancer associated with sarcoidosis -A case report-. Korean J Thorac Cardiovasc Surg 44: 301- 303, 2011.

14. Kyoraku Y, Ashitani J, Sakamoto A, Yanagi S, Matsumoto N and Nakazato M: A case of Hodgkin's lymphoma in a patient with sarcoidosis. Nihon Kokyuki Gakkai Zasshi 47: 900-905, 2009 (In Japanese).

15. Cherk MH, Pham A and Haydon A: 18F-fluorodeoxyglucose positron emission tomography-positive sarcoidosis after chemoradiotherapy for Hodgkin's disease: a case report. J Med Case Rep 29: 247, 2011 .

16. Kalff V, Hicks RJ, Ware RE, Hogg A, Binns D and McKenzie AF: The clinical impact of (18)F-FDG PET in patients with suspected or confirmed recurrence of colorectal cancer: a prospective study. J Nucl Med 43: 492-499, 2002.

17. Lequoy M, Coriat R, Rouquette A, et al: Sarcoidosis lung nodules in colorectal cancer follow-up: sarcoidosis or not? Am J Med 126: 642-645, 2013.

18. Choi JH, Shin JA, Park HK, et al: Sarcoidosis associated with oxaliplatin-based chemotherapy for colorectal cancer. Case Rep Oncol Med 2014: 203027, 2014.

19. Yao M, Funk GF, Goldstein DP, DeYoung BR and Graham MM: Benign lesions in cancer patients. Case 1. Sarcoidosis after chemoradiation for head and neck cancer. J Clin Oncol 23: 640-641, 2005

20. Arana Yi C, McCue P, Rosen M, Machtay M, Axelrod R and Morris GJ: Sarcoidosis mimicking metastatic bone disease in head and neck cancer. Semin Oncol 40: 529-534, 2013.

21. Hendrickx BW, van Herpen CM, Bonenkamp JJ, Bulten J and Oyen WJ: Positive positron emission tomography scan in sarcoidosis and two challenging cases of metastatic cancer. CASE 1. Mediastinal sarcoidosis in a melanoma patient treated with interferon. J Clin Oncol 23: 8906-8907, 2005 .
22. Wilgenhof S, Morlion V, Seghers AC, et al: Sarcoidosis in a patient with metastatic melanoma sequentially treated with anti-CTLA-4 monoclonal antibody and selective BRAF Inhibitor. Anticancer Res 32: 1355-1359, 2012.

23. Vogel WV, Guislain A, Kvistborg P, Schumacher TN, Haanen JB and Blank CU: Ipilimumab-induced sarcoidosis in a patient with metastatic melanoma undergoing complete remission. J Clin Oncol 30: e7-e10, 2012.

24. Kim MH, Lee K, Kim KU, Park HK, Lee MK and Suh DS: Sarcoidosis mimicking cancer metastasis following chemotherapy for ovarian cancer. Cancer Res Treat 45: 354-358, 2013.

25. Konishi H, Komatsu S, Ichikawa D, et al: Diagnostic problems in gastric cancer patients with sarcoidosis - case report and literature review. Gan To Kagaku Ryoho 39: 2330-2332, 2012.

26. Blank N, Lorenz HM, Ho AD and Witzens-Harig M: Sarcoidosis and the occurrence of malignant diseases. Rheumatol Int 2014 (Epub ahead of print).

27. Brincker $\mathrm{H}$ and Wilbek E: The incidence of malignant tumors in patients with sarcoidosis. Ugeskr Laeger 136: 2192-2195, 1974 (In Danish).

28. Yamaguchi M, Odaka M, Hosoda Y, Iwai K and Tachibana T: Excess death of lung cancer among sarcoidosis patients. Sarcoidosis 8: 51-55, 1991.

29. Askling J, Grunewald J, Eklund A, Hillerdal G and Ekbom A: Increased risk for cancer following sarcoidosis. Am J Respir Crit Care Med 160: 1668-1672, 1999.

30. Boffetta P, Rabkin CS and Gridley G: A cohort study of cancer among sarcoidosis patients. Int J Cancer 124: 2697-2700, 2009.

31. Ji J, Shu X, Li X, Sundquist K, Sundquist J and Hemminki K: Cancer risk in hospitalized sarcoidosis patients: a follow-up study in Sweden. Ann Oncol 20: 1121-1126, 2009.

32. Suen JS, Forse MS, Hyland RH and Chan CK: The malignancy-sarcoidosis syndrome. Chest 98: 1300-1302, 1990.

33. Rømer FK, Hommelgaard P and Schou G: Sarcoidosis and cancer revisited: a long-term follow-up study of 555 Danish sarcoidosis patients. Eur Respir J 12: 906-912, 1998.

34. Seersholm N, Vestbo J and Viskum K: Risk of malignant neoplasms in patients with pulmonary sarcoidosis. Thorax 52: 892-894, 1997. 